\title{
Coronary computed tomographic angiography (CCTA) in community hospitals: "current and emerging role"
}

This article was published in the following Dove Press journal:

Vascular Health and Risk Management

7 May 2010

Number of times this article has been viewed

\author{
Rakesh K Sharma' \\ Donald J Voelker' \\ Rajiv K Sharma' \\ Vibhuti N Singh ${ }^{2}$ \\ Girish Bhatt' \\ Mathilde Moazazi ${ }^{1}$ \\ Teresa Nash' \\ Hanumanth K Reddy' \\ 'Medical Center of South Arkansas, \\ El Dorado, University of Arkansas \\ for Medical Sciences, Little Rock, \\ AR, USA; ${ }^{2}$ Bayfront Medical Center \\ St Petersburg, University of South \\ Florida, FL, USA
}

\begin{abstract}
Coronary computed tomographic angiography (CCTA) is a rapidly evolving test for diagnosis of coronary artery disease. Although invasive coronary angiography is the gold standard for coronary artery disease (CAD), CCTA is an excellent noninvasive tool for evaluation of chest pain. There is ample evidence to support the cost-effective use of CCTA in the early triage process of patients presenting with chest pain in the emergency room. CCTA plays a critical role in the diagnosis of chest pain etiology as one of potentially fatal conditions, aortic dissection, pulmonary embolism, and myocardial infarction. This 'triple rule out' protocol is becoming an increasingly practicable and popular diagnostic tool in ERs across the country. In addition to a quick triage of chest pain patients, it may improve quality of care, decrease cost, and prevent medico-legal risk for missing potentially lethal conditions presenting as chest pain. CCTA is also helpful in the detection of subclinical and vulnerable coronary plaques. The major limitations for wide spread acceptance of this test include radiation exposure, motion artifacts, and its suboptimal imaging with increased body mass index.
\end{abstract}

Keywords: calcium scoring, computed tomography, coronary artery disease (CAD), angiography, coronary CTA, chest pain, community hospitals, emergency room, pulmonary embolism, aortic dissection

\section{Background}

Coronary computer tomographic angiography (CCTA) has been playing an incremental role in the diagnosis of coronary artery disease (CAD). CCTA has been especially useful in the triage of patients with acute chest pain. These patients are frequent visitors of emergency rooms posing an immediate diagnostic and therapeutic challenge. Chest pain patients present with potentially fatal conditions such as acute coronary syndrome (ACS), aortic dissection (AD), and pulmonary embolism (PE). Efficient evaluation of these chest pain patients with CCTA prevents complications, death, and disability. Evaluation and triage of more than 6,392,000 patients ${ }^{1}$ presenting with chest pain nationwide poses a significant challenge to the health care system and CCTA evaluation of these patients may alleviate this challenge and save not only lives, but also the cost and complications of invasive procedures such as cardiac catheterization. This becomes an important issue in small community hospitals where limited resources have to be efficiently used.

\section{Evolution of CCTA}

Since the pioneering work of Sir Godfrey N Hounsfield in 1972, CT technology has developed at a fast pace. Electron beam computed tomography (EBCT), otherwise called ultrafast CT or cine CT, was first introduced in 1980, followed by the 4-slice 
CT in the year 2000, 16-slice in 2002, and 64-slice in 2004. Initially cardiac CT, performed on 4-slice multidetector computed tomography (MDCT), examinations were confined to the evaluation of only proximal coronary arteries. There was poor spatial and temporal resolution with long acquisition time requiring intolerably long breath hold periods. This was revolutionized by the 16-slice MDCT, where for the first time a complete examination of coronary arteries was performed with a breath hold of just 20 seconds. Then the 64-slice MDCT scan became widely available. These scanners have much higher spatial and temporal resolution with a scan time of 12-15 seconds compared to 20 seconds with the 16-slice coronary CTA. This enables medical staff to perform a rapid screen for CAD in community hospitals.

The CT has undergone a dramatic improvement in imaging with evolution from a single-slice/detector to a multi-slice/detector leading to the commonly used terminology, MDCT or multi-slice computed tomography (MSCT). In this manuscript, for coronary imaging, the term CCTA will be used interchangeably with MDCT or MSCT.

This modality employs a rotating source of X-rays with circular and stationary detector arrays. X-ray photons are generated within a specialized X-ray tube mounted on a rotating gantry which pass through the patient's body and are detected by fixed rows of detector arrays, thus producing images of coronary arteries in seconds after the patient is scanned with current scanners as shown in Figures 1 and 2 .

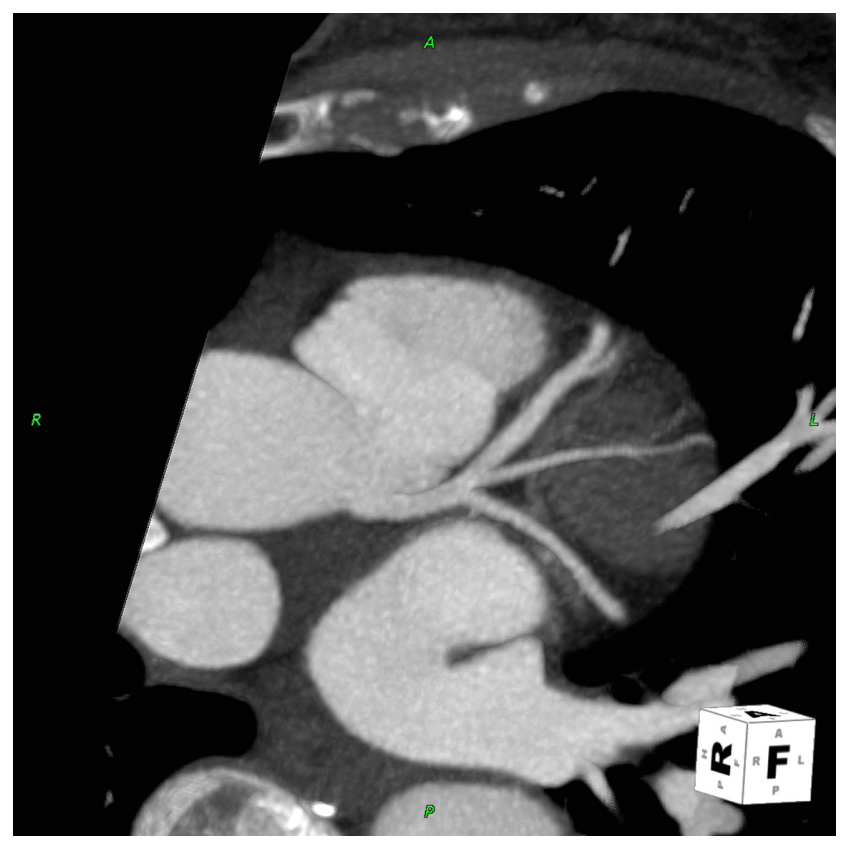

Figure I Illustration of thin MIP reconstruction of left coronary artery. Note: Similarity of CCTA image to an angiographic appearance.
Most recently, 256-slice and 320-slice scanners have been introduced. With this new generation of scanners, there has been a progressive improvement in spatial and temporal resolution. Similarly, dual-source CT (DSCT) scanners incorporate two tubes and two corresponding detectors which offer considerable advantage with temporal resolution as low as $83 \mathrm{~ms},{ }^{2}$ leading to elimination of a majority of artifacts. ${ }^{3}$ Its role is also evolving in the detection of subclinical atherosclerosis and vulnerable plaque, the most common cause of ACS.

\section{Scope of CCTA in patients with chest pain}

Chest pain is a frequent complaint among emergency room (ER) patients in this country. In many of these patients, basic evaluation by the ER physician including history, physical examination, electrocardiography (ECG), and initial cardiac biomarkers does not exhibit evidence of active myocardial ischemia. Inappropriate numbers of these patients are admitted to hospitals with suspected ACS leading to long hospital stays especially over the weekends. Extensive efforts have been made over the past decade to create chest pain centers with protocols in place for early detection, risk stratification, and timely discharge of chest pain patients. While this concept is helpful, there has been a continued practice of expensive evaluation and prolonged hospitalization for a majority of these patients presenting with chest pain.

More than $50 \%$ of ER admissions to the hospital are for observation to collect preliminary data to exclude ACS, PE, and AD. Unfortunately, positive yield of this large number

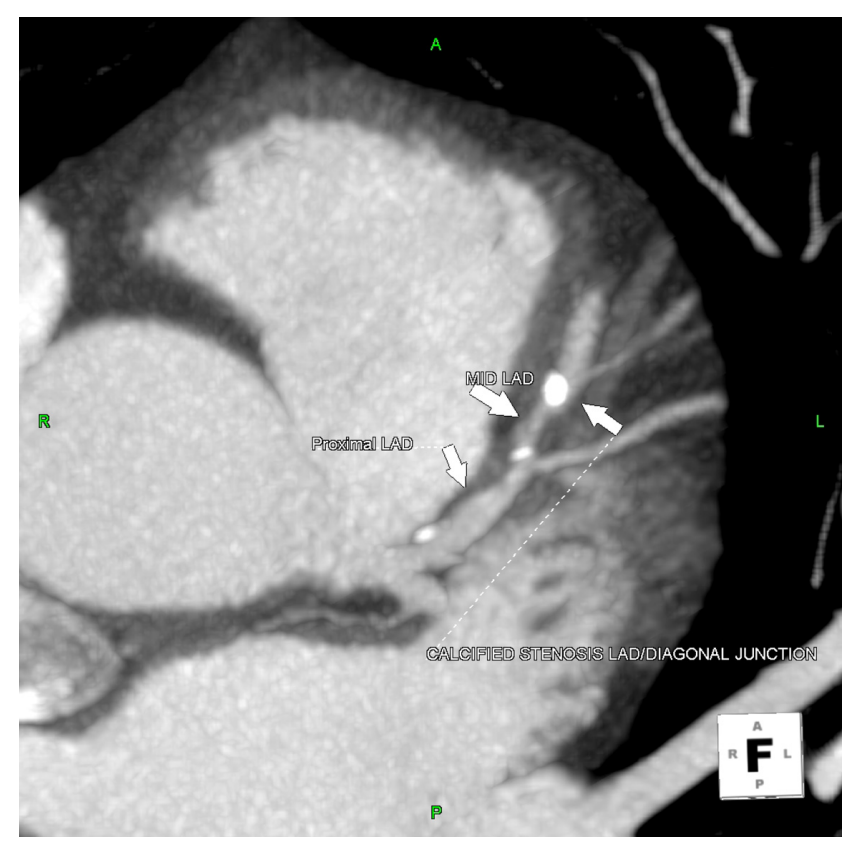

Figure 2 Illustration of soft and calcified plaque in proximal and mid LAD. 
of admissions is as low as $2 \%-5 \%, 4,5$ and a majority of these patients $(55 \%)$ are found to have noncardiac pathology. ${ }^{6}$ Conversely, $2 \%$ of patients with chest pain are discharged inappropriately with missed diagnosis of ACS, PE, and AD. ${ }^{6-8}$

The underlying reason for this practice is to exclude any potentially fatal etiologies of chest pain such as MI, PE, and AD. Therefore, physicians are reluctant to send these patients home from the ER because of the risk of $2 \%$ missed diagnosis of acute myocardial infarction (AMI) $)^{6,9,10}$ in these patients. This type of ER practice is fraught with a tremendous cost of more than 12 billion dollars a year. ${ }^{11}$ In community hospitals, the wide spread availability of CCTA as an important test may expedite early diagnosis and discharge of chest pain patients. Furthermore, unnecessary transfer of some of these patients to tertiary care hospitals may be avoided and significant health care savings may be achieved.

The gold standard for visualization of coronary arteries has been invasive coronary angiography. The use of this technique has increased many fold over the past three decades leading to exorbitant cost and inconvenience to the patients. ${ }^{12}$ Furthermore, it is plagued with the issue of operator dependent variability in coronary lesion assessment for percent stenosis. ${ }^{13}$ Consequently, there has been an increasing interest to develop a less invasive and cost effective technique for evaluation of coronary arteries. Out of many exciting technologies, CCTA is emerging as the most promising tool for the diagnosis of CAD, while excluding other potentially fatal conditions such as $\mathrm{AD}$ (Figure 3) and PE (Figure 4) which may also present with

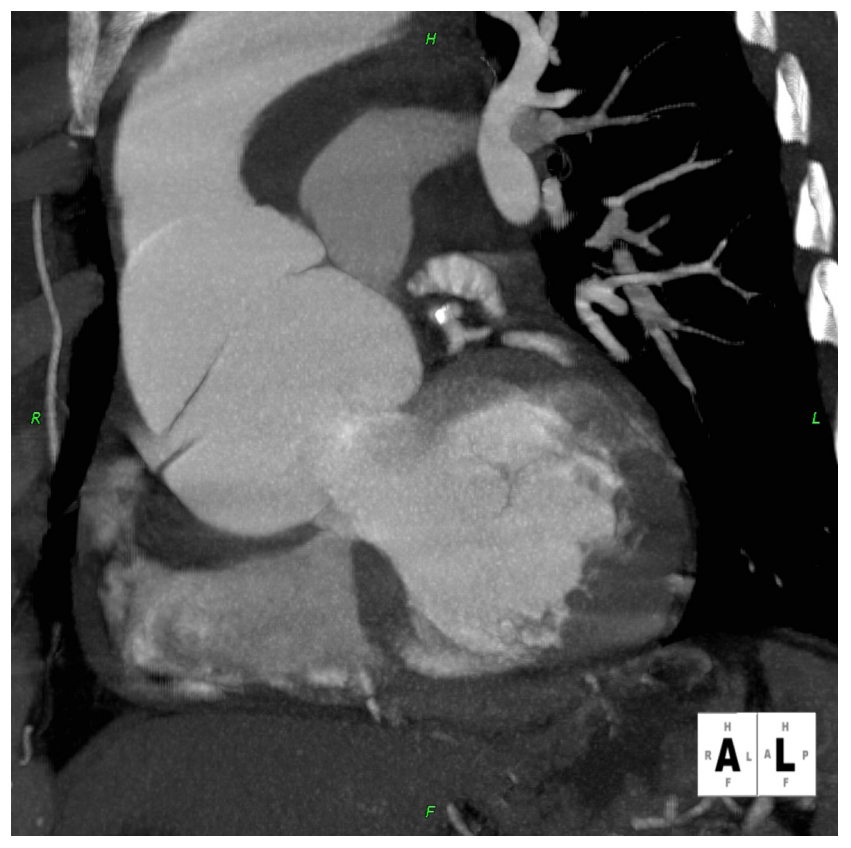

Figure 3 Patient presenting with acute chest pain; CCTA image showing large aortic root dissection. chest pain. Therefore, there is a great need for a noninvasive tool such as CCTA for definitive early diagnosis of the cause of chest pain in the ER.

\section{Current indications}

There is a definite role for CCTA in patients with chest pain who have low to intermediate probability of CAD and other cardiac conditions as listed in Table 1. There has been an emerging role for this test in the 'triple rule out' protocol for chest pain patients in the ER.

\section{Emerging indications}

The crucial role of CCTA for evaluation of chest pain and early triage of patients in the ER is evolving very fast. The feature of three dimensional volumetric acquisitions virtually allows unlimited views for image projection after processing. There has been intense interest in 'triple rule out' protocol for chest pain by a single acquisition that results in simultaneous opacification of coronary arteries, aorta, and arterial phase opacification of pulmonary arteries. This examination may need $110 \mathrm{cc}$ of contrast, a specialized post-processing with routine reconstruction for coronaries, wide field of view (FOV) coronal reconstruction of lungs, and oblique sagittal reconstruction for thoracic aorta. The major disadvantage of 'triple rule out' protocol is an increase in the radiation dose due to the large area of coverage and an increase in contrast use. CCTA has the advantage of faster acquisition in a single breath hold with excellent imaging resolution.

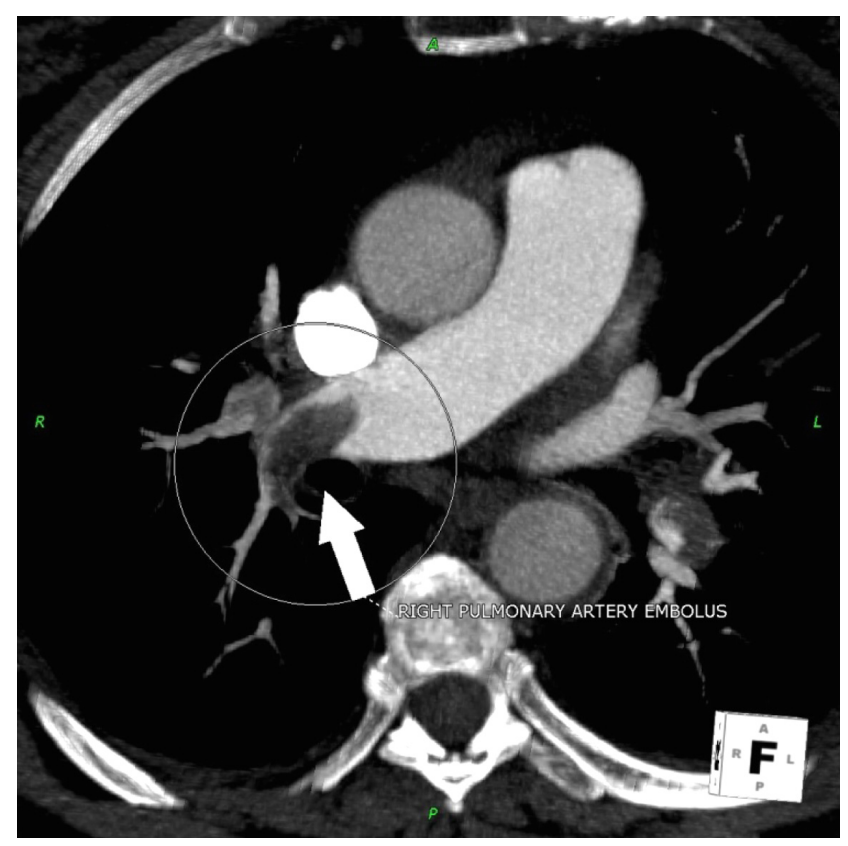

Figure 4 Patient presenting with acute chest pain; CCTA image showing large right pulmonary artery embolism. 
Table I Indications for CCTA

Chest pain syndrome:
- Intermediate pre-test probability
- Uninterpretable ECG
- Patients unable to exercise
- Detection of CAD with prior equivocal or uninterpretable test
Evaluation of CAD in new onset CHF
Noninvasive evaluation of LIMA prior to redo CABG
Assessment of complex congenital heart disease
Evaluation of cardiac masses
Evaluation of pericardial conditions
Patients with technically limited ECHO, MRI or TEE
Evaluation of pulmonary veins prior to AF ablation
Evaluation of coronary vein mapping prior to biventricular pacemaker
insertion
Evaluation of suspected aortic dissection and/or thoracic aortic
aneurysm
Evaluation of suspected pulmonary embolism

More importantly, widespread availability makes its usage feasible in community hospitals.

\section{Triage tools for chest pain}

There are several tools available in the ER for early triage of chest pain patients. The basic practice is to obtain history and physical examination (H\&P) with cardiac markers, chest X-ray, and ECG. H\&P is a crucial part, and therefore a majority of ERs have developed protocols to elicit all the pertinent history and physical findings. This basic practice helps to risk stratify the patients by TIMI score as illustrated in Table 2. The majority of these low to intermediate patients, ${ }^{14}$ with TIMI score less than three, do not have CAD, and due to very low yield in this group they end up getting admitted to the hospitals for observation and sequential cardiac biomarkers assessment prior to definite evaluation with a stress test and/or cardiac catheterization. Cardiac biomarkers are the standard of care and typically take 6-12 hours to become positive in the blood test and therefore many patients with active ischemia and/or injury may be missed by cardiac biomarkers criteria. CCTA in the ER as a triage tool provides a new paradigm shift, and its role is clearly and rapidly emerging.

There are many objective tests used in setting of the ER such as echocardiography, nuclear studies, magnetic resonance imaging (MRI), and coronary artery calcium (CAC) scoring. Echocardiography has a very limited role in chest pain evaluation due to its limited sensitivity for AMI in patients with ACS when pain has already resolved..$^{15,16}$ In late 1990, many chest pain centers developed the use
Table 2 TIMI score

\begin{tabular}{l}
\hline TIMI SCORE: (Assigned I point each) \\
\hline Medical history factors: \\
Age $>65$ \\
Known CAD with $>50 \%$ stenosis \\
Three or more coronary risk factors \\
(Positive FH, HTN, Hyperlipidemia, DM, Smoking) \\
Aspirin use in past 7 days \\
Clinical presentation factors: \\
Two or more angina events in 24 hours \\
Increase in cardiac markers \\
ST segment deviation
\end{tabular}

Abbreviations: $\mathrm{FH}$, family history; HTN, hypertension; DM, diabetes mellitus.

of the radionuclide stress test using single photo emission computed tomography (SPECT) to help risk stratification and early discharge of chest pain patients in the ER. Myocardial imaging studies with Tc-99m-based radioisotope have shown an excellent negative predictive value (NPV) as a screening test, ${ }^{17,18}$ though the positive predictive value may be limited due to suboptimal imaging resolution and artifacts. MRI has the ability to diagnose CAD and other potentially fatal conditions such as PE and AD. ${ }^{19,20}$ However, its use as a first line test is limited by multiple factors such as technical expertise, physician availability on site, and contraindications to MRI due to metallic implants.

Absence of CAC or minimal CAC predicted a very low incidence of future cardiac events in asymptomatic patients ${ }^{21-24}$ and symptomatic patients undergoing coronary angiography. ${ }^{6,9,10}$ Several studies have shown the value of CAC in ER patients with negative ECG and cardiac enzymes as a triage tool with a very high negative predictive value (NPV). ${ }^{25,26}$ Georgiou et al performed EBCT in 192 patients with chest pain and had an average follow up of $50 \pm 10$ months. Among this cohort, 30\% showed a graded relationship between all cardiac events and CAC score. ${ }^{26}$ This study showed CAC as a triage test with sensitivity of $97 \%$ and NPV of $99 \%$. Patients without CAC (CAC score $=0$ ) had 0.6/year future cardiovascular events. Recent studies have shown that CAC may be a useful tool in the ER for risk stratification of patients with ACS. Several other studies have demonstrated a significant correlation between $\mathrm{CAC}$ and the overall coronary artery atherosclerotic plaque burden. ${ }^{27-31}$ These studies have shown high sensitivity $>95 \%$ and high NPV of $>95 \%$.

\section{CCTA as a triage tool}

CCTA may become an attractive option as a triage tool in the ER due to its widespread availability and noninvasive 
approach. Several CCTA studies have shown an excellent correlation of CCTA with coronary angiography with sensitivities ranging from $92 \%-95 \%$ and NPV of $97 \%-98 \%$ for the diagnosis of significant CAD..$^{32-36}$ Two published meta-analyses have shown excellent sensitivity and specificity of CCTA for CAD as compared to coronary angiography. ${ }^{37,38}$ Moreover, CCTA has shown a promise in detecting noncalcified plaque, ${ }^{39}$ and an evaluation global left ventricular function comparable to MRI. ${ }^{40}$

As early as 1996, many studies have investigated its use as a triage tool in ER patients with chest pain, negative ECG, and cardiac enzymes. ${ }^{41}$ The NPV of CCTA in such patients has been reported to be $97 \%-100 \%$. Gallagher et al reported their experience with CCTA in low risk patients with negative ECG and cardiac markers in the $\mathrm{ER}^{42}$ where all patients had nuclear sestamibi scan and CCTA. This study reflected the accuracy of CCTA comparable to well established nuclear tests in these low risk patients.

Initial evaluation of ACS patients in the ER was performed using 16-slice CCTA by Kimura et al. ${ }^{43} \mathrm{~A}$ total of 74 consecutive patients with ACS (non-ST elevation) patients with ACS/non-ST elevation myocardial infarction (NSTEMI) underwent CCTA within 24 hours. Significant coronary lesions were correctly identified in 56 out of 57 patients. Similarly, 11 out of 13 patients without significant disease were correctly diagnosed. Lida et al have reported similar findings in 28 patients with ACS. ${ }^{44}$ Subsequently, there have been many studies using 64-slice CCTA. A single center experience in 70 patients using 64-slice CCTA showed sensitivity of $95 \%$, specificity of $90 \%$, and NPV of $93 \%$. ${ }^{36}$

A recent study, Rule Out Myocardial Infarction using Computer Assisted Tomography (ROMICAT) ${ }^{45}$ evaluated the value of CCTA in the triage of ER patients with ACS. This was an observational cohort study of 368 patients presenting to the ER with chest pain, normal troponin, and nonischemic ECG. 64-slice CCTA was performed in these patients before index hospitalization and results were not disclosed to treating physicians. End points were ACS during index hospitalization and major adverse cardiac events (MACE) in six months. Among this cohort, $50 \%$ of patients had no CAD on CCTA, 31\% had nonobstructive CAD, and only $19 \%$ had more than $50 \%$ stenosis or inconclusive considered as positive study. Moreover, door to CCTA was only 16 minutes in ROMICAT compared to 40.5 hours of an average hospital stay. ${ }^{46}$ Given these findings, a large number of patients did not need hospitalization and could have been triaged in the ER by CCTA.
This study and several other studies may support the use of CCTA in the ER. The ROMICAT study ${ }^{45}$ also demonstrated sensitivity and NPV of $100 \%$ for ACS. Several other studies have showed higher sensitivity, specificity, and NPV in evaluation of ACS in the ER. ${ }^{47-50}$ A recent presentation at the American Heart scientific session (2009) ${ }^{51}$ looked at the role of CCTA as a triage tool in the ER in patients presenting with chest pain in Computed Tomography for Systematic Triage of Acute chest pain and Treatment (CT-STAT) trial. In this trial, investigators included 749 patients presenting to the ER with acute chest pain within 12 hours of onset of symptoms with normal ECG and normal cardiac biomarkers. These patients were randomized to the CCTA arm or to the conventional standard of care treatment arm with myocardial perfusion imaging to expedite early diagnosis. In the CCTA arm, $82.3 \%$ had no significant disease leading to early discharge. Among the cohort undergoing conventional management, $90 \%$ had a normal myocardial perfusion study as part of standard work up. Early diagnosis (door to diagnosis) was much faster in the CCTA arm with mean diagnosis time 2.9 hours in the CCTA arm compared to 6.2 hours in the conventional standard of care arm with myocardial perfusion imaging. Moreover, the radiation exposure in the CCTA arm was $10.8 \mathrm{mSv}$ compared to $15 \mathrm{mSv}$ in the conventional arm. Overall cost in the CCTA arm was $\$ 2,138$ compared to $\$ 3,458$ in the conventional arm. This trial demonstrated the feasibility of effective triage of a chest pain patient with early door to diagnosis leading to early discharge with lesser radiation exposure. CCTA has great promise in detection of CAD in patients with a low pre-test probability and these constitute a large number of patients coming to the ER of community hospitals.

It may also be very useful in patients with atypical chest pain with left bundle branch block (LBBB) where frequent coronary angiography is performed to exclude CAD. Caussin et $\mathrm{l}^{52}$ evaluated the role of 64 -slice CCTA in 66 patients with LBBB and found a sensitivity of 97\%, specificity of $95 \%$, and NPV of $97 \%$ for detection of CAD with $50 \%$ stenosis. Similarly, it showed a $100 \%$ sensitivity and $92 \%$ specificity for detection of CAD in subsets of aortic stenosis (AS). ${ }^{53}$ Frequently, these patients with equivocal stress test or new onset of congestive heart failure (CHF) are admitted to the hospital for exclusion of significant CAD. CCTA combined with myocardial perfusion imaging has been increasingly used in intermediate risk patients. This allows simultaneous functional and anatomic assessment of CAD. ${ }^{54}$ Its role in assessing myocardial viability is being 
investigated to identify acute and old MI. ${ }^{55}$ With its spatial resolution, CCTA's emerging role is being explored in the assessment of aortic valve anatomy and pathology ${ }^{56,57}$ Other applications of CCTA include evaluation of pericardial and myocardial diseases as listed in Table 1.

\section{Role of CCTA in subclinical atherosclerosis (SCA)}

A recent study by Pundzuite et al showed that nonobstructive coronary disease on CCTA can lead to higher cardiovascular events as compared to patients without atherosclerotic plaque. ${ }^{58}$ Therefore, detection of sub-clinical atherosclerosis may lead to aggressive risk factor modification and a reduction in CAD burden of the general population. While this approach may save lives and money in the long term, immediate concerns are the expense of CCTA and the radiation issue. Meanwhile, $\mathrm{CAC}$ appears to be an excellent screening test for detection of sub-clinical CAD.

\section{Detection of vulnerable plaque}

The detection of vulnerable plaque leading to coronary events is of great interest. Detection of asymptomatic CAD has been debated since the publication of the Screening for Heart Attack Prevention and Education (SHAPE) Task Force report. ${ }^{59}$ Although invasive coronary angiography can grossly identify morphologic features such as luminal thrombosis, calcification, and plaque disruption, it fails to delineate qualitative features of the plaque predictive of rupture. ${ }^{60}$ While calcified plaque may be detected by CAC, the role of CCTA is evolving into the evaluation of soft plaque which may be the vulnerable plaque.

Small numbers of validation studies have demonstrated the accuracy of CCTA for imaging noncalcified plaque with sensitivity ranging from $80 \%-90 \% .{ }^{61-65}$ Certain indicators of vulnerable plaque, such as vessel remodeling or a high plaque volume noted on CCTA, may help predict ischemic events. ${ }^{65,66}$ Several small studies in ACS have found have a higher percentage of noncalcified plaque and positive remodeling in patients with ACS compared to stable angina. ${ }^{67-69}$ In most patients, AMI may be the first clinical manifestation of sub-clinical plaque rupture or erosion. ${ }^{70}$

There has been great debate in regard to imaging of this vulnerable plaque. Major technologies which can help image this plaque include intravascular ultrasound (IVUS), MRI, positron emission tomography (PET) imaging, and CCTA or MDCT. ${ }^{71}$ Increased carotid artery intimal thickness noted in ultrasound studies has been a marker of cardiovascular and cerebral atherosclerosis. ${ }^{72}$ Molecular imaging techniques have used radio-labeled molecules to detect the biological activity of plaques such as radioactive-labeled lipoproteins. ${ }^{73}$ IVUS of coronary plaques is useful in the evaluation of vulnerable plaques. CCTA has also been compared favorably with IVUS in the measurement of atherosclerotic plaque and lumen area ${ }^{74}$ CCTA certainly appears to be promising in the evaluation of plaque characteristics and associated arterial remodeling. ${ }^{75}$ Recently a prospective study demonstrated that patients with positively remodeled arteries with low attenuation plaques on $\mathrm{CT}$ angiography were at a higher risk of developing ACS in the subsequent follow up period. ${ }^{76}$

\section{Radiation issues}

With emerging indications of CCTA, there is concern about the long term effect of radiation. Current CT scan use for various studies is expected to contribute to a large number of future malignancies and it is estimated that 29,000 future cancers are possibly related to all CT scans done in $2007 .{ }^{77}$ Therefore, any radiation exposure should always be a concern for risk. One should familiarize oneself with the background radiation and the radiation associated with the commonly used tests in cardiology practice ${ }^{78-81}$ as shown in Table 3. Given this carcinogenic potential of radiation, health care workers who prescribe this radiation must be fully aware of radiation risks. They should have a full understanding of effective dose concept which is a standard of measure of exposure expressed in millisievert $(\mathrm{mSv})$. Effective dose is the sum of weighted equivalent doses in all the organs and tissues during a particular scan. Due to higher doses delivered to lungs and female breast in CCTA, there is a higher carcinogenic effect on these organs. This risk is higher in younger patients and more in women than

Table 3 Dose of radiation in different cardiac procedures

\begin{tabular}{ll}
\hline Examination & Mean effective dose \\
\hline Background radiation & $3 \mathrm{mSv} /$ year \\
Chest X-ray & $0.1 \mathrm{mSv}$ \\
Calcium scoring & $2 \mathrm{mSv}$ \\
Chest CT & $5-7 \mathrm{mSv}$ \\
CT abdomen and Pelvis & $8-11 \mathrm{mSv}$ \\
Coronary angiography & $5.6 \mathrm{mSv}$ \\
PTCA & $6.9 \mathrm{mSv}$ \\
Coronary angiography with PTCA & $9.3 \mathrm{mSv}$ \\
Coronary Angiography + PTCA + Stent & $13 \mathrm{mSv}$ \\
SPECT-MIBI & $11 \mathrm{mSv}$ \\
SPECT-Thallium & $25 \mathrm{mSv}$ \\
Coronary CTA (males) & $6.7-10.9 \mathrm{mSv}$ \\
Coronary CTA (Females) & $8.1-13.0 \mathrm{mSv}$ \\
\hline
\end{tabular}


in men. It is conceivable that in the near future patients may carry an "imaging card" reflecting the cumulative dose of radiation received by an individual patient which may be a factor to consider while ordering additional imaging studies requiring more radiation exposure.

Better scanners and software have contributed significantly to reduction in radiation exposure. Many dose reducing technical advances are available on 64-slice scans. These include cardiac filter modes, bowtie filters, and newer protocols such as ECG-triggered dose modulation and prospective gating. Quality control of scanners regarding the radiation issue is also very important. Recording of radiation dose for every patient in $\mathrm{mSv}$ should be mandatory and if there is a significant deviation from the published data, individual protocols in a given CCTA program should be reviewed. Government regulations via Environmental Protection Agency (EPA) require monitoring of credentials of ordering personnel, justified indications, and regular equipment check for minimum exposure and stringent indications for pregnant patients. For example, federal standards state that the radiation dose to an unborn child cannot exceed $5 \mathrm{mSv}$ over a nine-month period. This is because unborn children have rapidly growing cells and are therefore most sensitive to radiation.

\section{Preparation for CCTA program}

Meticulous preparation of the patient for CCTA is the key to obtaining a diagnostic image which would aid the management of patients with acute or chronic cardiac problems. Guidelines are listed in Table 4 and these may vary depending upon capabilities of the scanner such as single source or dual sources. ${ }^{82}$ As part of starting a CCTA program appropriate guidelines for documentation of competence ${ }^{83}$ should be

Table 4 General guidelines

Screen for contraindications

ECG rhythm and baseline HR

Assess renal function

Instructions and education

Proper placement of ECG leads

Administer $\beta$ Blockers: Individualize

- Oral 50-100 mg one hour before procedure

- IV-5 mg metoprolol prior to scan and practice breath holding

- Monitor HR during breath hold

- IV Metoprolol 5 mg q5 min x5 doses to target HR

- Calcium channel blocker if $\beta$-Blockers $\mathrm{Cl}$

Premedicate with S/L 0.4-0.8 mg NTG

Scan the patient delineated as a part of a quality control program. A scanner must be staffed by highly skilled and qualified technologists. Staff and physicians should be knowledgeable in the issues of radiation exposure, CT scan collimation, temporal resolution, and spatial resolution. Achieving a target heart rate of 60-70 is important for adequate images by a single source system. ${ }^{84}$ Pre-scan use of nitroglycerine is helpful for visualization of distal coronary arteries due to a $20 \%$ increase in the resultant diameter of coronary arteries. ${ }^{85}$

Quality control during acquisition of images is very important and staff should be knowledgeable of all the technical parameters such as table speed (pitch), window setting, level setting, reconstructions, post-processing, and image manipulations. A thorough understanding of contrast injection methods, adverse reactions, and contrast kinetics is critical. It is very important to understand how to overcome challenges of contrast-enhanced imaging of the left heart and surrounding structures. In high risk patients an iso-osmolar contrast such as Visipaque ${ }^{\circledR}$ should be selected as its viscosity is the same as blood, $290 \mathrm{~m}$ osmols $/ \mathrm{kg} \mathrm{H}_{2} 0$. All other contrast media have an osmolality of more than $600 \mathrm{~m}$ osmols $/ \mathrm{kg} \mathrm{H}_{2} 0$.

Instruction and education of the patient regarding CCTA is crucial in obtaining adequate images. Occasionally, simple obstacles such as the patient's inability to raise the arm above the head to move arms out the FOV or inability to hold their breath may be a relative contraindication for coronary imaging by CCTA. It is also crucial to exclude patients with known levels of high CAC score as CCTA images may be nonevaluable. ${ }^{86}$

\section{Conclusion}

The revolution of noninvasive imaging of the heart has taken a giant leap forward with widespread availability of the 64-slice CT, and now 256 and 320-slice CT scanners. The resultant superior spatial resolution, with improved temporal resolution with faster gantry rotation and dual source heads, offer the potential for remarkable improvement in sensitivity, specificity, and NPV in the diagnosis of CAD. Future generation scanners may provide an opportunity for first line imaging of CAD. Thus in patients presenting with chest pain, CCTA may be able to assess coronary lumen of calcified or noncalcified plaque and further evaluate other life threatening conditions such as AD and PE. The 'triple rule out' examination holds promise for revolutionizing the imaging of patients with acute chest pain in the ER. With wide spread availability in community hospitals, CCTA is likely to be embraced as the noninvasive triage tool of choice 
in the ER. Furthermore, a significant improvement in quality of care, cost-effectiveness, and medico-legal liability may be accomplished with future CCTA use in the ER. The key to implementation of such CCTA programs will be 'central reading stations' for expert reading and advice to the ER physicians, available 24 hours a day, year round for all the remote community hospitals just like night hawk radiology (Teleradiology).

\section{Disclosure}

The authors report no conflicts of interest in this work.

\section{References}

1. Pitts SR, Niska RW, Xu J, Burt CW. National Hospital Ambulatory Medical Care Survey: 2006 emergency department summary. National Health Statistics Reports. 2008;7:20.

2. Flohr TG, McCollough $\mathrm{CH}$, Bruder $\mathrm{H}$, et al. First performance evaluation of a dual-source CT (DSCT) system. Eur Radiol. 2006;16(2):256-268.

3. Dey D, Lee CJ, Ohba M, et al. Image quality and artifacts in coronary CT angiography with dual-source CT: initial clinical experience. J Cardiovasc Comput Tomogr. 2008;2(2):105-114.

4. Fineberg HV, Scadden D, Goldman L. Care of patients with a low probability of acute myocardial infarction. Cost effectiveness of alternatives to coronary-care-unit admission. $N$ Engl J Med. 1984;310(20):1301-1307.

5. McCarthy BD, Wong JB, Selker HP. Detecting acute cardiac ischemia in the emergency department: a review of the literature. J Gen Intern Med. 1990;5(4):365-373.

6. Pope JH, Aufderheide TP, Ruthazer R, et al. Missed diagnoses of acute cardiac ischemia in the emergency department. $N$ Engl J Med. 2000;342(16):1163-1170.

7. Lee TH, Rouan GW, Weisberg MC, et al. Clinical characteristics and natural history of patients with acute myocardial infarction sent home from the emergency room. Am J Cardiol. 1987;60(4):219-224.

8. McCarthy BD, Beshansky JR, D'Agostino RB, Selker HP. Missed diagnoses of acute myocardial infarction in the emergency department: results from a multicenter study. Ann Emerg Med. 1993;22(3):579-582.

9. Tatum JL, Jesse RL, Kontos MC, et al. Comprehensive strategy for the evaluation and triage of the chest pain patient. Ann Emerg Med. 1997;29(1):116-125.

10. Reilly BM, Evans AT, Schaider JJ, et al. Impact of a clinical decision rule on hospital triage of patients with suspected acute cardiac ischemia in the emergency department. JAMA. 2002;288(3):342-350.

11. Selker HP, Zalenski RJ, Antman EM, et al. An evaluation of technologies for identifying acute cardiac ischemia in the emergency department: a report from a National Heart Attack Alert Program Working Group. Ann Emerg Med. 1997;29(1):13-87.

12. Gershlick AH, de Belder M, Chambers J, et al. Role of non-invasive imaging in the management of coronary artery disease: an assessment of likely change over the next 10 years. A report from the British Cardiovascular Society Working Group. Heart. 2007;93(4):423-431.

13. Fleming RM, Kirkeeide RL, Smalling RW, Gould KL. Patterns in visual interpretation of coronary arteriograms as detected by quantitative coronary arteriography. J Am Coll Cardiol. 1991;18(4):945-951.

14. Antman EM, Cohen M, Bernink PJ, et al. The TIMI risk score for unstable angina/non-ST elevation MI: A method for prognostication and therapeutic decision making. JAMA. 2000;284(7):835-842.

15. Peels CH, Visser CA, Kupper AJ, Visser FC, Roos JP. Usefulness of two-dimensional echocardiography for immediate detection of myocardial ischemia in the emergency room. Am J Cardiol. 1990;65(11): 687-691.
16. Sabia P, Afrookteh A, Touchstone DA, Keller MW, Esquivel L, Kaul S. Value of regional wall motion abnormality in the emergency room diagnosis of acute myocardial infarction. A prospective study using two-dimensional echocardiography. Circulation. 1991; 84(3 Suppl):I85-92.

17. Heller GV, Stowers SA, Hendel RC, et al. Clinical value of acute rest technetium-99m tetrofosmin tomographic myocardial perfusion imaging in patients with acute chest pain and non-diagnostic electrocardiograms. J Am Coll Cardiol. 1998;31(5):1011-1017.

18. Udelson JE, Beshansky JR, Ballin DS, et al. Myocardial perfusion imaging for evaluation and triage of patients with suspected acute cardiac ischemia: a randomized controlled trial. JAMA. 2002;288(21): 2693-2700.

19. Arpasi PJ, Bis KG, Shetty AN, White RD, Simonetti OP. MR angiography of the thoracic aorta with an electrocardiographically triggered breath-hold contrast-enhanced sequence. Radiographics. 2000;20(1):107-120.

20. Kwong RY, Schussheim AE, Rekhraj S, et al. Detecting acute coronary syndrome in the emergency department with cardiac magnetic resonance imaging. Circulation. 2003;107(4):531-537.

21. O'Malley PG, Taylor AJ, Jackson JL, Doherty TM, Detrano RC. Prognostic value of coronary electron-beam computed tomography for coronary heart disease events in asymptomatic populations. Am J Cardiol. 2000;85(8):945-948.

22. Raggi P, Callister TQ, Cooil B, et al. Identification of patients at increased risk of first unheralded acute myocardial infarction by electron-beam computed tomography. Circulation. 2000;101(8):850-855.

23. Wong ND, Hsu JC, Detrano RC, Diamond G, Eisenberg H, Gardin JM. Coronary artery calcium evaluation by electron beam computed tomography and its relation to new cardiovascular events. Am J Cardiol. 2000;86(5):495-498.

24. Arad Y, Spadaro LA, Goodman K, Newstein D, Guerci AD. Prediction of coronary events with electron beam computed tomography. $J \mathrm{Am}$ Coll Cardiol. 2000;36(4):1253-1260.

25. McLaughlin VV, Balogh T, Rich S. Utility of electron beam computed tomography to stratify patients presenting to the emergency room with chest pain. Am J Cardiol. 1999;84(3):327-328, A328.

26. Georgiou D, Budoff MJ, Kaufer E, Kennedy JM, Lu B, Brundage BH. Screening patients with chest pain in the emergency department using electron beam tomography: a follow-up study. $J$ Am Coll Cardiol. 2001;38(1):105-110.

27. Simons DB, Schwartz RS, Edwards WD, Sheedy PF, Breen JF, Rumberger JA. Noninvasive definition of anatomic coronary artery disease by ultrafast computed tomographic scanning: a quantitative pathologic comparison study. J Am Coll Cardiol. 1992;20(5):1118-1126.

28. Rumberger JA, Schwartz RS, Simons DB, Sheedy PF 3rd, Edwards WD, Fitzpatrick LA. Relation of coronary calcium determined by electron beam computed tomography and lumen narrowing determined by autopsy. Am J Cardiol. 1994;73(16):1169-1173.

29. Kaufmann RB, Peyser PA, Sheedy PF, Rumberger JA, Schwartz RS. Quantification of coronary artery calcium by electron beam computed tomography for determination of severity of angiographic coronary artery disease in younger patients. $J$ Am Coll Cardiol. 1995;25(3):626-632.

30. Rumberger JA, Simons DB, Fitzpatrick LA, Sheedy PF, Schwartz RS. Coronary artery calcium area by electron-beam computed tomography and coronary atherosclerotic plaque area. A histopathologic correlative study. Circulation. 1995;92(8):2157-2162.

31. Rumberger JA, Sheedy PF 3rd, Breen JF, Schwartz RS. Coronary calcium, as determined by electron beam computed tomography, and coronary disease on arteriogram. Effect of patient's sex on diagnosis. Circulation. 1995;91(5):1363-1367.

32. Mollet NR, Cademartiri F, Nieman K, et al. Multislice spiral computed tomography coronary angiography in patients with stable angina pectoris. J Am Coll Cardiol. 2004;43(12):2265-2270.

33. Nieman K, Cademartiri F, Lemos PA, Raaijmakers R, Pattynama PM, de Feyter PJ. Reliable noninvasive coronary angiography with fast submillimeter multislice spiral computed tomography. Circulation. 2002;106(16):2051-2054. 
34. Ropers D, Baum U, Pohle K, et al. Detection of coronary artery stenoses with thin-slice multi-detector row spiral computed tomography and multiplanar reconstruction. Circulation. 2003;107(5):664-666.

35. Martuscelli E, Romagnoli A, D'Eliseo A, et al. Accuracy of thin-slice computed tomography in the detection of coronary stenoses. Eur Heart J. 2004;25(12):1043-1048

36. Raff GL, Gallagher MJ, O’Neill WW, Goldstein JA. Diagnostic accuracy of noninvasive coronary angiography using 64-slice spiral computed tomography. J Am Coll Cardiol. 2005;46(3):552-557.

37. Vanhoenacker PK, Heijenbrok-Kal MH, Van Heste R, et al. Diagnostic performance of multi-detector $\mathrm{CT}$ angiography for assessment of coronary artery disease: meta-analysis. Radiology. 2007;244(2):419-428.

38. Sun Z. Diagnostic accuracy of multislice CT angiography in peripheral arterial disease. J Vasc Interv Radiol. 2006;17(12):1915-1921.

39. Schoenhagen P, White RD, Nissen SE, Tuzcu EM. Coronary imaging: angiography shows the stenosis, but IVUS, CT, and MRI show the plaque. Cleve Clin J Med. 2003;70(8):713-719.

40. Halliburton SS, Petersilka M, Schvartzman PR, Obuchowski N, White RD. Evaluation of left ventricular dysfunction using multiphasic reconstructions of coronary multi-slice computed tomography data in patients with chronic ischemic heart disease: validation against cine magnetic resonance imaging. Int J Cardiovasc Imaging. 2003;19(1):73-83.

41. Budoff MJ, Georgiou D, Brody A, et al. Ultrafast computed tomography as a diagnostic modality in the detection of coronary artery disease: a multicenter study. Circulation. 1996;93(5):898-904.

42. Gallagher MJ, Ross MA, Raff GL, Goldstein JA, O’Neill WW, O’Neil B. The diagnostic accuracy of 64-slice computed tomography coronary angiography compared with stress nuclear imaging in emergency department low-risk chest pain patients. Ann Emerg Med. 2007;49(2):125-136.

43. Kimura S, Kakuta T, Kuboyama O, et al. Multisclice computed tomography for risk stratification in patients with suspected Non ST elevation acute coronary syndrome [abstract]. Circulation. 2004;110 Supp III

44. Lida K, Sata Y, Matsumoto N. Usefulness of multislice computed tomography to identify acute coronary in the emergency department [abstract]. Circulation. 2004;110 Supp III.

45. Hoffmann U, Bamberg F, Chae CU, et al. Coronary computed tomography angiography for early triage of patients with acute chest pain: the ROMICAT (Rule Out Myocardial Infarction using Computer Assisted Tomography) trial. J Am Coll Cardiol. 2009;53(18):1642-1650.

46. Hlatky MA. Evaluating use of coronary computed tomography angiography in the emergency department. $\mathrm{J} \mathrm{Am} \mathrm{Coll} \mathrm{Cardiol.}$ 2009;53(18):1651-1652.

47. White CS, Kuo D, Kelemen M, et al. Chest pain evaluation in the emergency department: can MDCT provide a comprehensive evaluation? AJR Am J Roentgenol. 2005;185(2):533-540.

48. Sato Y, Matsumoto N, Ichikawa M, et al. Efficacy of multislice computed tomography for the detection of acute coronary syndrome in the emergency department. Circ J. 2005;69(9):1047-1051.

49. Hoffmann U, Nagurney JT, Moselewski F, et al. Coronary multidetector computed tomography in the assessment of patients with acute chest pain. Circulation. 2006;114(21):2251-2260.

50. White C, Read K, Kuo D. Assessment of chest pain in the emergency room: what is the role of multidetector CT? Eur J Radiol. 2006;57(3):368-372

51. American Heart Association newsroom [online]. [cited 2009, Nov 18]. Available from: http://americanheart.mediaroom.com/index. php?s=54\&item $=217$

52. Caussin C, Larchez C, Ghostine S, et al. Comparison of coronary minimal lumen area quantification by 64-slice computed tomography versus intravascular ultrasound for intermediate stenosis. Am J Cardiol. 2006;98(7):871-876

53. Meijboom WB, Mollet NR, Van Mieghem CA, et al. Pre-operative computed tomography coronary angiography to detect significant coronary artery disease in patients referred for cardiac valve surgery. J Am Coll Cardiol. 2006;48(8):1658-1665.
54. Danciu SC, Herrera CJ, Stecy PJ, Carell E, Saltiel F, Hines JL. Usefulness of multislice computed tomographic coronary angiography to identify patients with abnormal myocardial perfusion stress in whom diagnostic catheterization may be safely avoided. Am J Cardiol. 2007;100(11):1605-1608.

55. Lardo AC, Cordeiro MA, Silva C, et al. Contrast-enhanced multidetector computed tomography viability imaging after myocardial infarction: characterization of myocyte death, microvascular obstruction, and chronic scar. Circulation. 2006;113(3):394-404.

56. Pannu HK, Jacobs JE, Lai S, Fishman EK. Gated cardiac imaging of the aortic valve on 64-slice multidetector row computed tomography: preliminary observations. J Comput Assist Tomogr. 2006;30(3): $443-446$.

57. Gilkeson RC, Markowitz AH, Balgude A, Sachs PB. MDCT evaluation of aortic valvular disease. AJR Am J Roentgenol. 2006;186(2):350-360.

58. Pundziute G, Schuijf JD, Jukema JW, et al. Prognostic value of multislice computed tomography coronary angiography in patients with known or suspected coronary artery disease. J Am Coll Cardiol. 2007;49(1):62-70

59. Naghavi M, Falk E, Hecht HS, et al. From vulnerable plaque to vulnerable patient - part III: executive summary of the Screening for Heart Attack Prevention and Education (SHAPE) Task Force report. Am J Cardiol. 2006;98(2A):2H-15H.

60. Falk E, Shah PK, Fuster V. Coronary plaque disruption. Circulation. 1995;92(3):657-671.

61. Becker CR, Knez A, Ohnesorge B, Schoepf UJ, Reiser MF. Imaging of noncalcified coronary plaques using helical CT with retrospective ECG gating. AJR Am J Roentgenol. 2000;175(2):423-424.

62. Schoenhagen P, Tuzcu EM, Stillman AE, et al. Non-invasive assessment of plaque morphology and remodeling in mildly stenotic coronary segments: comparison of 16-slice computed tomography and intravascular ultrasound. Coron Artery Dis. 2003;14(6):459-462.

63. Leber AW, Knez A, Becker A, et al. Accuracy of multi-detector spiral computed tomography in identifying and differentiating the composition of coronary atherosclerotic plaques: a comparative study with intracoronary ultrasound. J Am Coll Cardiol. 2004;43(7): 1241-1247.

64. Achenbach S, Moselewski F, Ropers D, et al. Detection of calcified and noncalcified coronary atherosclerotic plaque by contrastenhanced, submillimeter multi-detector spiral computed tomography: a segment-based comparison with intravascular ultrasound. Circulation. 2004;109(1):14-17.

65. Achenbach S, Ropers D, Hoffmann U, et al. Assessment of coronary remodeling in stenotic and nonstenotic coronary atherosclerotic lesions by multi-detector spiral computed tomography. J Am Coll Cardiol. 2004;43(5):842-847.

66. Leber AW, Becker A, Knez A, et al. Accuracy of 64-slice computed tomography to classify and quantify plaque volumes in the proximal coronary system: a comparative study using intravascular ultrasound. J Am Coll Cardiol. 2006;47(3):672-677.

67. Leber AW, Knez A, White CW, et al. Composition of coronary atherosclerotic plaques in patients with acute myocardial infarction and stable angina pectoris determined by contrast-enhanced multislice computed tomography. Am J Cardiol. 2003;91(6):714-718.

68. Inoue F, Sato Y, Matsumoto N, Tani S, Uchiyama T. Evaluation of plaque texture by means of multislice computed tomography in patients with acute coronary syndrome and stable angina. Circ J. 2004;68(9):840-844.

69. Hoffmann U, Moselewski F, Nieman K, et al. Noninvasive assessment of plaque morphology and composition in culprit and stable lesions in acute coronary syndrome and stable lesions in stable angina by multidetector computed tomography. J Am Coll Cardiol. 2006;47(8): $1655-1662$.

70. Virmani R, Burke AP, Farb A, Kolodgie FD. Pathology of the vulnerable plaque. J Am Coll Cardiol. 2006;47(8 Suppl):C13-C18.

71. Chen JW, Wasserman BA. Vulnerable plaque imaging. Neuroimaging Clin NAm. 2005;15(3):609-621, xi. 
72. O'Leary DH, Polak JF. Intima-media thickness: a tool for atherosclerosis imaging and event prediction. Am J Cardiol. 2002;90(10C): $18 \mathrm{~L}-21 \mathrm{~L}$.

73. Langer HF, Haubner R, Pichler BJ, Gawaz M. Radionuclide imaging: a molecular key to the atherosclerotic plaque. J Am Coll Cardiol. 2008;52(1):1-12.

74. Leber AW, Knez A, von Ziegler F, et al. Quantification of obstructive and non-obstructive coronary lesions by 64-slice computed tomography: a comparative study with quantitative coronary angiography and intravascular ultrasound. J Am Coll Cardiol. 2005;46(1):147-154.

75. Achenbach S. Can $\mathrm{CT}$ detect the vulnerable coronary plaque? Int J Cardiovasc Imaging. 2008;24(3):311-312.

76. Motoyama S, Sarai M, Harigaya H, et al. Computed tomographic angiography characteristics of atherosclerotic plaques subsequently resulting in acute coronary syndrome. J Am Coll Cardiol. 2009;54(1): 49-57.

77. Berrington de Gonzalez A, Mahesh M, Kim KP, et al. Projected cancer risks from computed tomographic scans performed in the United States in 2007. Arch Intern Med. 2009;169(22):2071-2077.

78. Hunold P, Vogt FM, Schmermund A, et al. Radiation exposure during cardiac CT: effective doses at multi-detector row CT and electron-beam CT. Radiology. 2003;226(1):145-152.

79. Cury RC, Feutchner G, Pena CS, Janowitz WR, Katzen BT, Ziffer JA. Acute chest pain imaging in the emergency department with cardiac computed tomography angiography. J Nucl Cardiol. 2008;15(4): $564-575$.
80. Betsou S, Efstathopoulos EP, Katritsis D, Faulkner K, Panayiotakis G. Patient radiation doses during cardiac catheterization procedures. $\mathrm{BrJ}$ Radiol. 1998;71(846):634-639.

81. Morin RL, Gerber TC, McCollough CH. Radiation dose in computed tomography of the heart. Circulation. 2003;107(6):917-922.

82. Halliburton SS, Abbara S. Practical tips and tricks in cardiovascular computed tomography: patient preparation for optimization of cardiovascular CT data acquisition. J Cardiovasc Comput Tomogr. 2007;1(1):62-65.

83. Budoff MJ, Cohen MC, Garcia MJ, et al. ACCF/AHA clinical competence statement on cardiac imaging with computed tomography and magnetic resonance: a report of the American College of Cardiology Foundation/American Heart Association/American College of Physicians Task Force on Clinical Competence and Training. J Am Coll Cardiol. 2005;46(2):383-402.

84. Giesler T, Baum U, Ropers D, et al. Noninvasive visualization of coronary arteries using contrast-enhanced multidetector CT: influence of heart rate on image quality and stenosis detection. AJR Am J Roentgenol. 2002;179(4):911-916.

85. Dewey M, Hoffmann H, Hamm B. Multislice CT coronary angiography: effect of sublingual nitroglycerine on the diameter of coronary arteries. Rofo. 2006;178(6):600-604.

86. Ong TK, Chin SP, Liew CK, et al. Accuracy of 64-row multidetector computed tomography in detecting coronary artery disease in 134 symptomatic patients: influence of calcification. Am Heart J. 2006;151(6):1323 e1321-1326.
Vascular Health and Risk Management

\section{Publish your work in this journal}

Vascular Health and Risk Management is an international, peerreviewed journal of therapeutics and risk management, focusing on concise rapid reporting of clinical studies on the processes involved in the maintenance of vascular health; the monitoring, prevention and treatment of vascular disease and its sequelae; and the involvement of

\section{Dovepress}

metabolic disorders, particularly diabetes. This journal is indexed on PubMed Central and MedLine. The manuscript management system is completely online and includes a very quick and fair peer-review system, which is all easy to use. Visit http://www.dovepress.com/ testimonials.php to read real quotes from published authors. 